\title{
Subject Index Vol. 211, 1997
}

Abdominal wall abscess 308 Abducens palsy 109 Active retinitis 95 Acute esotropia 109

Additivity 354 Age-related macular degeneration 115

- $\quad$ maculopathy 283Agriculture 251 Amacrine cells 161Angiotensin II type 1 receptor

384 Angiotensinogen 301 Anterior cerebral artery 256

chamber 93

chamber-associated immune deviation 84

uveitis 305 Antibodies, protozoan 87 Antimetabolites 344 Aotes trivirgatus 201 Aphakia 21

Apraclonidine 354 Aqueous humor 84,380

- concentrations 229 Atopic dermatitis 263 Atropine 57

Axial length 271

Behçet's disease 266 Birdlike tapered fingertips

263 Blood mercury 402

- $\quad$ pressure 325, 327Blue field entoptic phenomenon 172

Body weight 325 Botulinum toxin A 387 Bovine eye 153 Bromocriptine mesylate 397 Brush

cytology 1,358 Bumetanide 153

Carotid-cavernous fistula 53 Cataract 242, 327, 394

surgery 75, 79, 292 Cavernous hemangioma 320

sinus thrombosis 308 Cefuroxime axetil 229 Cell culture 126

Central retinal vein occlusion 13,397

Chick 4 Chordoma 109 Chorioretinitis 87 Choroid 161

Choroidal detachment 53 Ciliary muscle 201 Clindamycin 95 Color vision test 25 Computer reconstruction 183 Confocal microscopy 147,183 Congenital toxoplasmosis 95 Conjunctiva 296

Contact lenses 32 Contractility 153 Contrast sensitivity 214

- test 214 Corneal endothelium 32

thickness 341 Corneoscleral lamellar kerato-

plasty 367 Craftsmanship 251 Craniopharyngioma 256 Creeping angle closure 205 Crozafon-De Laage punch 72 Cyclopentolate 57 Cycloplegia 57 Cynomolgus monkey 201 Cystoid macular edema 236 Cytokines 223

Dermoid, congenital benign

367 Diagnosis, differential 87 Dilated pupil 205 Diplopia 109 DNA strand breaks 192 Drug instillation test 332 Dry eye 358 Dyskeratosis, conjunctival 101

\section{ECCE 8}

Eccrine adenocarcinoma 44 Echography 61 Electron microscopy 296 ELISA method 226

Endoscopy 283 Eosinophils 1 Epidemiology 251 Epidermal growth factor 226 Ethacrynic acid 153 Excimer laser 32 Experimental glaucoma 178

Extracellular material 140

- $\quad$ matrix 183Eye infection 87Eyelid tumor 40

Fat suppression 362 
Filtering surgery 93

Fixed pupil 205

Flat anterior chamber 61

Flavobacterium indologenes 98

Flufenamic acid 153

Fluorescein angiography 115

Fluorometholone 394

Fluoropyrimidines 344

5-Fluorouridine 344

Focal retinochoroidal scar 95

Ganglion cells 161 Gaze palsy 320 Genetic defects 126 Glaucoma 21,25,72,79,93, 147, $172,288,327,338,354$

filtering surgery 61 Glucocorticoids 126 Glutathione peroxidase 192

reductase 192 Goggles 4

Gradient index endoscopes 283 Ground water 98

Hang-back recession 373

Hemodialysis 325

Heparin surface modification

75 High-resolution observation

283 HLA system 266 Holmium:YAG laser 274 Household dust 1 Human vitreous 226 Hydrogen peroxide 192 Hyperopia 274

IgG anü-Toxoplasma antibody

95 Image analysis 140 Immersion scleral shells 61 Immunohistochemistry 44,351 Independent toes 263 Indian file pattern 362 Indocyanine green angiography

115

Industry 251 Interleukin-la 49 Interleukin-1ß 49 Interleukin-8 223 Intracranial extension 236

Intraocular epithelial invasion 312

lens $75,79,214$

- implantation 332

pressure $79,172,325,354$

Iris cyst 312

Ischemic central retinal vein occlusion 13

Japanese cedar pollen 1 Juvenile cataract 263

- glaucoma 140

Kelly Descemet membrane

punch 93 Keratitis 98

Labeled microspheres 178 Lacrimal sac tumor 44 Lamina cribrosa 183 Laser iridotomy 205

- $\quad$ photocoagulation 13

thermal keratoplasty 274 Lens epithelium 192

extraction 247

opacity 242 Leukocyte velocity 172 Levator palpebrae superioris

muscle 387 Light 4

microscopy 183,296

reflex 332 Lighting 25 Limbus 367 Liposomes 344 Local anesthesia 327 LOCSII 242

Lymphocytes 1

Macacafascicularis 201 Macroaneurysm 115 Macular edema 13 
- $\quad$ hole 247

Magnetic resonance imaging

308 Meibomian gland 40

KAHGEIl

E-Mail karger@karger.ch Fax+4161306 1234 http://www.karger.ch

(C) 1997 S. KargerAG, Basel

407

Trabecular meshwork 126,140,

147,153 Trabeculectomy 93 Trabeculo-indentation 21 Transforming growth factor- $\alpha$

226 - - - factor-ß 380 Transgenic mice 192 Trauma 399 Traumatic iris cyst 312 Tuberculoid leprosy 305 Type 1 diabetes 209

Upper eyelid retraction 387 Uveitis 87

Vancomycin 232 Vasculature 161 Visual acuity 242

axis 391

deprivation 4

evoked potential 172

field(s) 256, 338

- defects 104

- loss 66 Vitamin A 101,358 Vitrectomy 219,247,399 Vitreous 223

cyst 391

fluorophotometry 209

hemorrhage 219 Vogt-Koyanagi-Harada disease

316

Work accidents 251

Metastatic eyelid carcinoma

362 Methicillin-resistant staphylo-

coccus 232 Metoclopramide 292 Miosis 332

Miotic-resistant pupil 205 Mites 1

Mitomycin C 93, 373 Monkey 161 mRNA expression 301

- $\quad$ ocular tissues 384Myopia 4,66,271

NADPH diaphorase 201 Natural history 90 Nerve cell 201

fiber analyzer 338

- layer 338 Neutrophils 1 Non-contact tonometry 341 Nonischemic central retinal

vein occlusion 13 Nonselective cation channel

153 Nosocomial infection 98

Ocular blood flow 178

pulse 271 Ondansetron 292 Ophthalmoplegia 320 Opportunistic infection 98 Optic chiasm 104 nerve head 183 Orbit 236

Orbital foreign body 402 Overlap syndrome 266 Owl monkey 201 Oxidative stress 126

Papillophlebitis 266 Paramedian pontine reticular

formation 320 Parameter estimation and

dosage adjustment program

232

Perfused anterior segment 153

Peripheral anterior synechiae 205 
Phacoemulsification 8,72

-, aspiration 79, 327, 332

Phagocytosis 147

Pharmacology 126

Photorefractive keratectomy 32,341,380,394

Pilocarpine 354

Pituitary adenoma 104

Pons 320

Posterior scleritis 316

Postoperative nausea and vomiting 292

Primary angle-closure glaucoma 205

- $\quad$ open-angle glaucoma 66Proliferative diabetic retinopathy 219

- $\quad$ vitreoretinopathy 49, 223,344

Prospective study 288 Pseudotumor 236 Psoriatic arthritis 266 Pulsatile ocular blood flow 271

Pupil dynamics 332 Pupillary dilator muscle 332

- $\quad$ sphincter muscle 332Purtscher's retinopathy 268

Quality control 75

Rabbit model 373 Rabbits 49 Rat 296

ocular tissues 301 Refractive error 242,271 Retina 268,351,399 Retinal circulation 172

detachment 223

-, late recurrent 247

toxicity 344

vasculitis 266

vein occlusion 288

vessels 268

Retinoblastoma 351

Retinotomy 399

Retrobulbar block 327

Reverse-transcription poly-merase chain reaction 301,384

Rhegmatogenous retinal detachment 90

Rheumatoid factor 316

Risk factor 66

S-100 protein 351 Sawchuck-Zaske equation 232 Scanning electron microscopy 183

- $\quad$ laser ophthalmoscope 115,268

Scleral buckling 112 Sebaceous carcinoma 40 Secondary glaucoma 305 Silicone oil 219

Smoking 296 Sodium fluorescein 209 Spondyloarthritis 266 Steroid-induced glaucoma

140 Subretinal hemorrhage 399

- $\quad$ lesions 112

- $\quad$ surgery 283 Superficial punctate keratitis

305

T helper lymphocyte 84

- $\quad$ suppressor lymphocyte 84Tamoxifen therapy 362Thermokeratoplasty 274Thermometer 402ThinPrep ${ }^{\circledR}$ process 358 Thyroid-associated eye disease

387 Timolol 354 Tissue plasminogen activator

399 Tobacco 296 Topical anesthesia 327 Toxoplasmosis, ocular 87 
408

O.Vol. Ophthalmologica Vol. 211,1997

Subject Index 